of carbon. Hayes and Goulds report a counting efficiency of 60-70 per cent for carbon-14 with the diphenyloxazole-toluene phosphor, and it should therefore be possible to realize at least 50 per cent efficiency after addition of acetylene, even if the 23 per cent reduction observed in our experiments is not compensated by increase in amplification.

At $-78^{\circ} \mathrm{C}$. the thermal noise from the photomultipliers will be substantiolly reduced, and with a coincidence circuit the reduction in the random noise count will be proportional to the square of the reduction factor for a single tube. Further measurements using tubes in coincidence are in progress and will be reported elsewhere.

B. N. AUDRIC

J. V. P. LONG

Chemical Research Laboratory,

Teddington, Middlesex. March 2.

1 Hayes, Williams and Rogers, Los Alamos Report LADC. 1397 (March 1953).

${ }^{2}$ Arnold, Science, 119, 155 (1954).

Crathorn, Nature, 172, 632 (1953).

- Barker, Nature, 172, 631 (1953).

'Hayes and Grould, Science, 117, 482 (1953).

\section{Crystal Structure of Parabanic Acid}

A PReCrse investigation of the crystal structure of parabanic acid has been undertaken to obtain accurate bond-lengths and bond-angles for comparison with the crystal structures of diketopiperazine ${ }^{1}$ and cyanuric acid2, and with its infra-red spectrum. Crystals of parabanic acid were easily obtained by slowly cooling a hot, concentrated water solution. X-ray examination showed that the crystals belong to the monoclinio system with space group $P 2_{1} / n$, and the unit cell dimensions are: $a=10 \cdot 684$, $b=8 \cdot 194, c=5 \cdot 044$ A. $; \beta=92^{\circ} 30^{\prime}$. There are four molecules in the unit cell.

Three-dimensional intensity data were obtained from equi-inclination Weissenberg photographs. Multiple films were used, and the intensities were measured by visual comparison with an intensity scale.

Approximate positions of the atoms were obtained from a three-dimensional Patterson function, and have been refined from two-dimensional Fourier series and by three-dimensional least-squares treatment. During a late stage in the refinement of the positional parameters, a difference synthesis was computed, and this showed that the oxygen atoms had markedly anisotropic temperature factors which were very different from those of the other atoms. In order to correct for this, and to see whether this correction would affect the positional parameters, complete anisotropic temperature factors for each atom were introduced. They have the form :

$$
\exp -\left(\alpha h^{2}+\beta k^{2}+\gamma l^{2}+\delta h k+\varepsilon h l+\eta k l\right) .
$$

These temperature factors were then refined twice by the method of least squares. A final simultaneous refinement of positional parameters and temperature factors led to calculated structure factors which were in good agreement with the observed values. The discrepancy factor $R=\frac{\Sigma\left|F_{0}-F_{c}\right|}{\Sigma\left|F_{0}\right|}$ is 0.094 . The changes in positional parameters which resulted from the introduction of the temperature factors were small, with an average value of $0.003 \mathrm{~A}$.

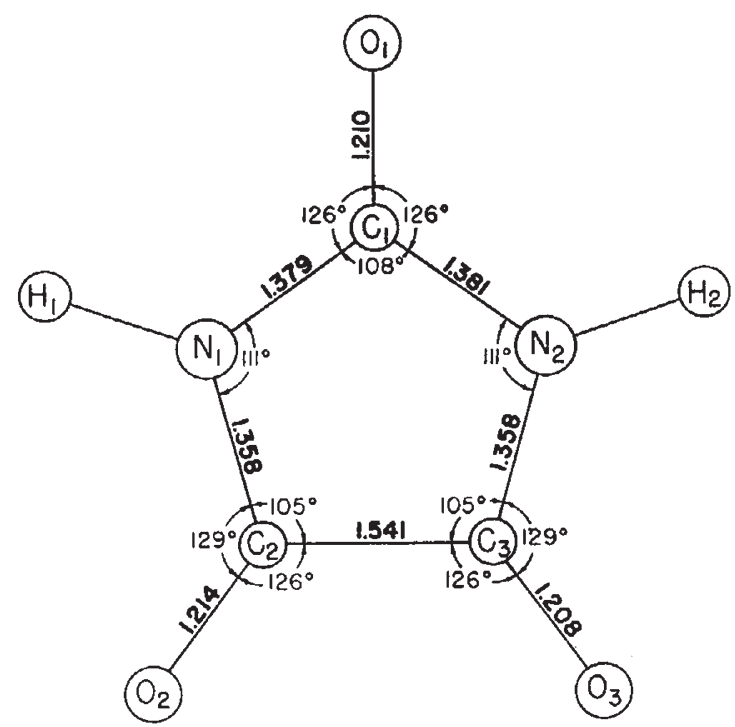

Fig. 1. The bond-lengths and bond-angles of parabanic acid

The structure consists of planar molecules inclined by $36^{\circ}$ to the $(010)$ plane. Neighbouring molecules related by the $n$ glide plane are linked in sheets by NH... O hydrogen bonds. The lengths of these bonds are $\mathrm{N}_{1} \ldots \mathrm{O}_{3^{\prime}}=2 \cdot 874 ; \mathrm{N}_{2} \ldots \mathrm{O}_{2^{\prime \prime}}=2 \cdot 835 \mathrm{~A}$. The oxygen of the unique carbonyl group is not involved in hydrogen bond formation. The bond-lengths and bond-angles of the molecule are shown in Fig. 1 .

A more detailed account of this work will be published elsewhere.

This investigation was supported in part by a research grant, $R G-1987$, from the National Institutes of Health, Public Health Service.

David R. Davies

California Institute of Technology, J. J. BLUM

Pasadena 4. Feb. 1 .

${ }^{1}$ Corey, R. B., J. Amer. Chem. Soc., 601598 (1938).
Wlebenga, E. H., and Moerman, N. F., Z. Krist., 99, 217 (1938).

\section{Direct Observation of Polygonization in Rocksalt}

Polygontzation has been studied hitherto by means of two different methods: Guinier and co-workers ${ }^{2}$ used refined X-ray methods, which, however, only permit the detection of orientation differences without localizing the domain responsible for a particular spot ; Cahn and others ${ }^{2}$, on the other hand, used etching techniques to make visible the polygon boundaries. The mean difference of orientation was estimated from the radius of curvature of the bent specimen and the width of the domains.

We describe here a further simple approach to the study of polygonization, based on the property of cleavage. When a crystal is polygonized, it is in fact to be expected that its cleavage faces will be poly. gonal. This has now been confirmed by observations on sodium chloride crystals. The method permits direct observation of the domain and an accurate measurement of the differences of orientation.

Konobejewski and Mirer ${ }^{3}$ have observed by means of the Laue method that bent crystals of sodium 